PROCEEDINGS OF THE

AMERICAN MATHEMATICAL SOCIETY

Volume 131, Number 12, Pages 3833-3837

S 0002-9939(03)07192-2

Article electronically published on July 9, 2003

\title{
A NOTE ON INVERTIBILITY PRESERVERS ON BANACH ALGEBRAS
}

\author{
MATEJ BREŠAR, AJDA FOŠNER, AND PETER ŠEMRL
}

(Communicated by David R. Larson)

\begin{abstract}
Let $\mathcal{A}$ be $\mathcal{B}$ be semisimple Banach algebras and let $\phi: \mathcal{A} \rightarrow \mathcal{B}$ be a unital bijective linear operator that preserves invertibility. If the socle of $\mathcal{A}$ is an essential ideal of $\mathcal{A}$, then $\phi$ is a Jordan isomorphism.
\end{abstract}

\section{INTRODUCTION}

Let $\mathcal{A}$ and $\mathcal{B}$ be algebras with identity elements, and let $\phi: \mathcal{A} \rightarrow \mathcal{B}$ be a linear map. We say that $\phi$ is unital if it maps the identity element of $\mathcal{A}$ into the identity element of $\mathcal{B}$, and we say that $\phi$ preserves invertibility if $\phi(x)$ is invertible in $\mathcal{B}$ whenever $x$ is invertible in $\mathcal{A}$. It turns out that, under rather mild assumptions, Jordan homomorphisms are unital invertibility preserving maps (see e.g. [10] Proposition 1.3]). Motivated by various relevant results (such as the Gleason-Kahane-Żelazko theorem) Kaplansky [9] asked when the converse is true, that is, under which assumptions a unital invertibility preserving map must be a Jordan homomorphism. There has been a lot of activity concerning this question; we refer the reader to some rather recent papers $([3,[4,6],[10])$ for historical accounts. We shall now only briefly discuss those results that are closely connected with the present paper.

By $\mathcal{B}(X)$ we denote the algebra of all bounded linear operators on a Banach space $X$. In 8 Jafarian and Sourour proved that Jordan isomorphisms are the only bijective unital linear operators between $\mathcal{B}(X)$ and $\mathcal{B}(Y)$ that preserve invertibility in both directions (i.e., $x$ is invertible if and only if $\phi(x)$ is invertible). Aupetit and du Mouton [4] extended this result to semisimple Banach algebras whose socle is an essential ideal (actually, they considered a slightly more general problem on maps preserving the full spectrum of each element). Finally, Sourour [10] showed that the result from $[8]$ is true for maps that preserve invertibility (in only one direction).

The goal of this note is to obtain results similar to those in [4], however, under the assumption that the invertibility is preserved in one direction only. In particular, we shall thereby obtain a brief proof of Sourour's result [10]. It should be mentioned, however, that several ideas from both [4] and [10] will be used in our proof.

By a Banach algebra we shall mean a complex Banach algebra with an identity element. The socle of the algebra $\mathcal{A}$ will be denoted by $\operatorname{soc}(\mathcal{A})$. Recall that an ideal $\mathcal{I}$ of $\mathcal{A}$ is said to be essential if it has a nonzero intersection with every nonzero

Received by the editors July 25, 2002.

2000 Mathematics Subject Classification. Primary 46H05, 46H10, 47B48.

Partially supported by a grant from the Ministry of Science of Slovenia.

(C)2003 American Mathematical Society 
ideal of $\mathcal{A}$; in semisimple algebras this is equivalent to the condition that $a \cdot \mathcal{I}=0$, where $a \in \mathcal{A}$, implies $a=0$.

We are now in a position to state our main result, which extends [4, Theorem 3.2 and Corollary 3.3].

Theorem 1.1. Let $\mathcal{A}$ and $\mathcal{B}$ be semisimple Banach algebras and let $\phi: \mathcal{A} \rightarrow \mathcal{B}$ be a unital bijective linear operator that preserves invertibility. Then

$$
\phi^{-1}\left(\phi\left(a^{2}\right)-\phi(a)^{2}\right) \cdot \operatorname{soc}(\mathcal{A})=0 \quad \text { for every } a \in \mathcal{A} .
$$

In particular, if $\operatorname{soc}(\mathcal{A})$ is an essential ideal of $\mathcal{A}$, then $\phi$ is a Jordan isomorphism.

In primitive algebras every nonzero ideal is essential, and from a well-known theorem of Herstein [7] on Jordan homomorphisms onto prime rings it follows easily that every Jordan isomorphism $\phi$ from a primitive algebra onto another algebra is either an isomorphism or an anti-isomorphism (just consider $\phi^{-1}$ ). Hence we have the following corollary, which generalizes [10, Main theorem].

Corollary 1.2. Let $\mathcal{A}$ be a primitive Banach algebra with nonzero socle, and let $\mathcal{B}$ be a semisimple Banach algebra. If $\phi: \mathcal{A} \rightarrow \mathcal{B}$ is a unital bijective linear operator that preserves invertibility, then $\phi$ is either an isomorphism or an anti-isomorphism.

\section{Proof}

We first fix the notation and terminology. By $\mathcal{A}$ and $\mathcal{B}$ we denote semisimple Banach algebras, and by $\phi$ a bijective unital linear operator from $\mathcal{A}$ onto $\mathcal{B}$ that preserves invertibility. Note that the latter assumption can be equivalently formulated as $\sigma(\phi(a)) \subseteq \sigma(a)$ for every $a \in \mathcal{A}$, where $\sigma(x)$ denotes the spectrum of the element $x$.

Recall that every minimal left ideal of $\mathcal{A}$ is of the form $\mathcal{A} e$ where $e$ is a minimal idempotent, i.e., $e^{2}=e \neq 0$ and $e \mathcal{A} e=\mathbb{C} e$. In this case $e \mathcal{A}$ is a minimal right ideal of $\mathcal{A}$. The sum of all minimal left ideals of $\mathcal{A}$ is called the socle of $\mathcal{A}$ and it coincides with the sum of all minimal right ideals of $\mathcal{A}$. For example, for any Banach space $X, \operatorname{soc}(\mathcal{B}(X))$ is equal to the ideal of all finite rank operators in $\mathcal{B}(X)$. If $\mathcal{A}$ has no minimal one-sided ideals, then we define $\operatorname{soc}(\mathcal{A})=0$. We say that a nonzero element $u \in \mathcal{A}$ has rank one if $u$ belongs to some minimal left ideal of $\mathcal{A}$ (equivalently, $u=u e$ for some minimal idempotent $e$ in $\mathcal{A}$ ). By $\mathcal{F}_{1}(\mathcal{A})$ we denote the set of all elements of rank one in $\mathcal{A}$. It is easy to see (see [5] for details) that $u \in \mathcal{F}_{1}(\mathcal{A})$ if and only if $u \neq 0$ and $u$ lies in some minimal right ideal of $\mathcal{A}$, and furthermore, this is equivalent to the condition that $u \mathcal{A} u=\mathbb{C} u \neq 0$. Another, less obvious characterization is that $u \in \mathcal{F}_{1}(\mathcal{A})$ if and only if $u \neq 0$ and $|\sigma(z u) \backslash\{0\}| \leq 1$ for every $z \in \mathcal{A}$ or, equivalently, $|\sigma(u z) \backslash\{0\}| \leq 1$ for every $z \in \mathcal{A}$ (see [4] or [5]).

Lemma 2.1. $\phi\left(\mathcal{F}_{1}(\mathcal{A})\right) \subseteq \mathcal{F}_{1}(\mathcal{B})$.

Proof. Pick $u \in \mathcal{F}_{1}(\mathcal{A})$. We have to show that $v=\phi(u)$ lies in $\mathcal{F}_{1}(\mathcal{B})$, that is, that $|\sigma(z v) \backslash\{0\}| \leq 1$ for every $z \in \mathcal{B}$.

From a well-known result of Aupetit [1] (see also [2, Theorem 5.5.2]) it follows that $\phi$ is continuous, and therefore of course $\phi^{-1}$ is also continuous. Set $M=$ $\left(2\left\|\phi^{-1}\right\|+1\right)^{-1}$ and pick $z \in \mathcal{B}$ such that $\|z\| \leq M$. Since $M<1,1+z$ is invertible, and we have $y=(1+z)^{-1}-1=-z+z^{2}-z^{3}+z^{4}-\cdots$. Set $x=\phi^{-1}(y)$ and note that

$$
\|x\| \leq\left\|\phi^{-1}\right\|\|y\| \leq\left\|\phi^{-1}\right\| \frac{\|z\|}{1-\|z\|} \leq\left\|\phi^{-1}\right\| \frac{M}{1-M}=\frac{1}{2},
$$


so that $1+x$ is invertible, whence it follows that $1+x-\lambda u=(1+x)\left(1-\lambda(1+x)^{-1} u\right)$ is invertible for all but possibly one $\lambda \in \mathbb{C}$. Since $\phi$ preserves invertibility, the same is true for

$$
\phi(1+x-\lambda u)=1+y-\lambda v=(1+y)(1-\lambda(1+z) v)
$$

which means that the spectrum of $(1+z) v$ contains at most one nonzero point. Thus we proved that $|\sigma((1+z) v) \backslash\{0\}| \leq 1$ whenever $\|z\| \leq M$, and similarly we see that in this case also $|\sigma(v(1+z)) \backslash\{0\}| \leq 1$.

Now let $z \in \mathcal{B}$ be any element. Define the analytic function $f: \mathbb{C} \rightarrow \mathcal{B}$ by $f(\lambda)=(\lambda+z) v$ and note that $|\sigma(f(\lambda)) \backslash\{0\}| \leq 1$ whenever $|\lambda|>\frac{\|z\|}{M}$.

Suppose that $v$ does not have a left inverse. Since, in particular, $|\sigma(f(\lambda))| \leq 2$ whenever $|\lambda|>\frac{\|z\|}{M}$, it follows from [2, Theorem 3.4.25] that $|\sigma(f(\lambda))| \leq 2$ for every $\lambda \in \mathbb{C}$. Taking $\lambda=0$ we thus get $|\sigma(z v)| \leq 2$. However, since $0 \in \sigma(z v)$, it follows that $|\sigma(z v) \backslash\{0\}| \leq 1$, as desired. The case when $v$ does not have a right inverse can be treated similarly, by considering the function $\lambda \mapsto v(\lambda+z)$. So we may assume that $v$ is invertible. In this case $|\sigma(f(\lambda))|=1$ whenever $|\lambda|>\frac{\|z\|}{M}$, and so applying [2, Theorem 3.4.25] again we see that this holds true for any $\lambda \in \mathbb{C}$. Accordingly, $|\sigma(z v)|=1$, and so, in particular, $|\sigma(z v) \backslash\{0\}| \leq 1$ (incidentally we mention that in the case when $v$ is invertible we actually have $\mathcal{A} \cong \mathcal{B} \cong \mathbb{C}$ ).

Given $u \in \mathcal{F}_{1}(\mathcal{A})$, there is $\tau(u) \in \mathbb{C}$ such that $u^{2}=\tau(u) u$. Clearly $\tau(u) \in \sigma(u)$, and moreover, either $\tau(u)=0$ or $\tau(u)$ is the only nonzero point in $\sigma(u)$. Since $\tau(u)$ is unique, we may consider $\tau$ as a function from $\mathcal{F}_{1}(\mathcal{A})$ to $\mathbb{C}$, and we extend it by defining $\tau(0)=0$. Using $u \mathcal{A} u=\mathbb{C} u, u \in F_{1}(\mathcal{A})$, and considering $(x u)^{2}$ and $(u x)^{2}$ it follows easily that $\tau(x u) u=u x u=\tau(u x) u$ for any $x \in \mathcal{A}$. Furthermore, we claim that $\tau\left(x_{1} u+x_{2} u\right)=\tau\left(x_{1} u\right)+\tau\left(x_{2} u\right)$ for all $x_{1}, x_{2} \in \mathcal{A}$ and $u \in \mathcal{F}_{1}(\mathcal{A})$. This follows from [4, Lemma 2.3], but it can also be proved using only elementary tools. Indeed, examining $\left(x_{1} u+x_{2} u\right)^{2}=x_{1} u x_{1} u+x_{1} u x_{2} u+x_{2} u x_{1} u+x_{2} u x_{2} u$ and applying $u x u=\tau(x u) u$ we get

$$
\left(\tau\left(x_{1} u+x_{2} u\right)-\tau\left(x_{1} u\right)-\tau\left(x_{2} u\right)\right)\left(x_{1} u+x_{2} u\right)=0,
$$

from which our assertion can be easily inferred. Also, it is straightforward to check that $\tau(\lambda u)=\lambda \tau(u)$ for all $\lambda \in \mathbb{C}$ and $u \in \mathcal{F}_{1}(\mathcal{A})$. Therefore, the restriction of $\tau$ to any minimal left ideal $\mathcal{A} u$ is a linear functional. Moreover, from $u^{2}=\tau(u) u$, $u \in \mathcal{F}_{1}(\mathcal{A})$, we conclude that $|\tau(u)| \leq\|u\|$ and so $\tau$ is bounded on $\mathcal{A} u$.

Lemma 2.2. $\tau(x u)=\tau(\phi(x) \phi(u))$ and $\tau\left(x^{2} u\right)=\tau\left(\phi(x)^{2} \phi(u)\right)$ for all $x \in \mathcal{A}$ and $u \in \mathcal{F}_{1}(\mathcal{A})$.

Proof. Let $u \in \mathcal{F}_{1}(\mathcal{A})$ be a fixed element. Pick a nonzero $x \in \mathcal{A}$ and let $D_{x}=\{\lambda \in$ $\left.\mathbb{C}|| \lambda \mid<(\|\phi\|\|x\|)^{-1}\right\}$. Then $1-\lambda \phi(x)$ is invertible for every $\lambda \in D_{x}$; moreover, since $\|\phi\| \geq 1$ ( $\phi$ is unital!), the same is true for $1-\lambda x$. We have $\phi(u) \in \mathcal{F}_{1}(\mathcal{B})$ and so we can define $F_{x}, G_{x}: D_{x} \rightarrow \mathbb{C}$ by

$$
F_{x}(\lambda)=\tau\left((1-\lambda x)^{-1} u\right), \quad G_{x}(\lambda)=\tau\left((1-\lambda \phi(x))^{-1} \phi(u)\right) .
$$

Since $\tau$ is a continuous linear functional on $\mathcal{A} u$ (resp. $\mathcal{B} \phi(u)$ ), we have

$$
F_{x}(\lambda)=\sum_{k=0}^{\infty} \tau\left(x^{k} u\right) \lambda^{k}, \quad G_{x}(\lambda)=\sum_{k=0}^{\infty} \tau\left(\phi(x)^{k} \phi(u)\right) \lambda^{k} .
$$

Suppose that $G_{x}(\lambda)=\alpha \neq 0$ for some $\lambda \in D_{x}$. Then $(1-\lambda \phi(x))^{-1} \phi(u)-\alpha$ is not invertible, and hence also $\phi(u)-\alpha(1-\lambda \phi(x))$ is not invertible. Since $\phi$ is 
unital and preserves invertibility, it follows that $u-\alpha(1-\lambda x)$ is not invertible. Accordingly, $(1-\lambda x)^{-1} u-\alpha$ is not invertible, which means that $F_{x}(\lambda)=\alpha$. That is, we showed that $G_{x}(\lambda)=F_{x}(\lambda)$ whenever $G_{x}(\lambda) \neq 0$. Since $F_{x}$ and $G_{x}$ are analytic functions, it follows that either $F_{x} \equiv G_{x}$ or $G_{x} \equiv 0$.

Comparing coefficients at the expansions of $F_{x}$ and $G_{x}$ we see, in particular, that for any $x \neq 0$ in $\mathcal{A}$ we have either $\tau(\phi(x) \phi(u))=0$ or $\tau(x u)=\tau(\phi(x) \phi(u))$ and $\tau\left(x^{2} u\right)=\tau\left(\phi(x)^{2} \phi(u)\right)$. Both conditions are trivially satisfied for $x=0$.

If $\tau(\phi(x) \phi(u))=0$ for all $x \in \mathcal{A}$, then we would have $\phi(u) \phi(x) \phi(u)=0$ for every $x \in \mathcal{A}$. However, since $\phi$ is onto and $\mathcal{B}$ is semisimple (and so, in particular, semiprime) this would yield $\phi(u)=0$, a contradiction. Thus $\tau\left(\phi\left(x_{1}\right) \phi(u)\right) \neq 0$ for some $x_{1} \in \mathcal{A}$. Then of course $\tau\left(x_{1}^{2} u\right)=\tau\left(\phi\left(x_{1}\right)^{2} \phi(u)\right)$. Now suppose there exists $x_{2} \in \mathcal{A}$ such that $\tau\left(x_{2}^{2} u\right) \neq \tau\left(\phi\left(x_{2}\right)^{2} \phi(u)\right)$. Then $\tau\left(\phi\left(x_{2}\right) \phi(u)\right)=0$; hence $\tau\left(\phi\left(x_{1}+\mu x_{2}\right) \phi(u)\right) \neq 0$ for any $\mu \in \mathbb{C}$, which in turn implies that $\tau\left(\left(x_{1}+\mu x_{2}\right)^{2} u\right)=$ $\tau\left(\phi\left(x_{1}+\mu x_{2}\right)^{2} \phi(u)\right)$. That is,

$$
\begin{aligned}
\mu\left(\tau\left(x_{1} x_{2} u+x_{2} x_{1} u\right)-\tau\left(\phi\left(x_{1}\right) \phi\left(x_{2}\right) \phi(u)\right.\right. & \left.\left.+\phi\left(x_{2}\right) \phi\left(x_{1}\right) \phi(u)\right)\right) \\
& +\mu^{2}\left(\tau\left(x_{2}^{2} u\right)-\tau\left(\phi\left(x_{2}\right)^{2} \phi(u)\right)\right)=0
\end{aligned}
$$

for every $\mu \in \mathbb{C}$, which clearly contradicts our assumption that $\tau\left(x_{2}^{2} u\right) \neq$ $\tau\left(\phi\left(x_{2}\right)^{2} \phi(u)\right)$. This means that $\tau\left(x^{2} u\right)=\tau\left(\phi(x)^{2} \phi(u)\right)$ for every $x \in \mathcal{A}$. In a similar (but of course shorter) fashion one shows that also $\tau(x u)=\tau(\phi(x) \phi(u))$ for every $x \in \mathcal{A}$.

Proof of Theorem 1.1. Let $x \in \mathcal{A}$ and let $u \in \mathcal{F}_{1}(\mathcal{A})$. From the first identity in Lemma 2.2 we see that $\tau\left(x^{2} u\right)=\tau\left(\phi\left(x^{2}\right) \phi(u)\right)$ and from the second one we see that $\tau\left(x^{2} u\right)=\tau\left(\phi(x)^{2} \phi(u)\right)$. Comparing we get $\tau\left(\left(\phi\left(x^{2}\right)-\phi(x)^{2}\right) \phi(u)\right)=0$. Set $x_{0}=\phi^{-1}\left(\phi\left(x^{2}\right)-\phi(x)^{2}\right)$, and note that $\tau\left(x_{0} u\right)=\tau\left(\phi\left(x_{0}\right) \phi(u)\right)=0$ for every $u \in \mathcal{F}_{1}(\mathcal{A})$. But this yields that $x_{0} u=0$ for every $u \in \mathcal{F}_{1}(\mathcal{A})$. Indeed, if $x_{0} u_{0}$ was not 0 for some $u_{0} \in \mathcal{F}_{1}(\mathcal{A})$, then, by the semisimplicity of $\mathcal{A}$, there would be $x \in \mathcal{A}$ such that $\sigma\left(x_{0} u_{0} \cdot x\right) \neq\{0\}$, meaning that $\tau\left(x_{0} \cdot u_{0} x\right) \neq 0$, a contradiction. Accordingly, $x_{0} \cdot \operatorname{soc}(\mathcal{A})=0$.

\section{REFERENCES}

[1] B. Aupetit, The uniqueness of the complete norm topology in Banach algebras and Banach Jordan algebras, J. Funct. Anal. 47 (1982), 1-6. MR 83g:46044

[2] B. Aupetit, "A primer on spectral theory", Springer-Verlag, New York, 1991. MR 92c:46001

[3] B. Aupetit, Spectrum-preserving linear mappings between Banach algebras or Jordan-Banach algebras, J. London Math. Soc. (2) 62 (2000), 917-924. MR 2001h:46078

[4] B. Aupetit and H. du Mouton, Spectrum preserving linear mappings in Banach algebras, Studia Math. 109 (1994), 91-100. MR 95c:46070

[5] M. Brešar and P. Šemrl, Finite rank elements in semisimple Banach algebras, Studia Math. 128 (1998), 287-298. MR 99a:46089

[6] M. Brešar and P. Šemrl, Spectral characterization of idempotents and invertibility preserving linear maps, Expo. Math. 17 (1999), 185-192. MR 2000d:16050

[7] I. N. Herstein, Jordan homomorphisms, Trans. Amer. Math. Soc. 81 (1956), 331-341. MR 17:938t

[8] A. A. Jafarian and A. R. Suorour, Spectrum preserving linear maps, J. Funct. Anal. 66 (1986), 255-261. MR 87m:47011

[9] I. Kaplansky, "Algebraic and analytic aspects of operator algebras", Regional Conference Series in Mathematics 1, Amer. Math. Soc., Providence, RI, 1970. MR 47:845 
[10] A. R. Sourour, Invertibility preserving linear maps on $\mathcal{L}(X)$, Trans. Amer. Math. Soc. 348 (1996), 13-30. MR 96f:47069

Department of Mathematics, University of Maribor, PF, KorošKa 160, SI-2000 MariBOR, SLOVENIA

E-mail address: bresar@uni-mb.si

Department of Mathematics, University of Maribor, PF, Koroška 160, Si-2000 MariBOR, SLOVENIA

E-mail address: ajda.fosner@uni-mb.si

Department of Mathematics, University of Ljubljana, Jadranska 19, SI-1000 LjublJANa, Slovenia

E-mail address: peter.semrl@fmf.uni-lj.si 